\title{
Robust Pulse Rate Variability Analysis from Reflection and Transmission Photoplethysmographic Signals
}

\author{
Elena Peralta ${ }^{1}$, Jesús Lázaro ${ }^{2}$, Eduardo Gil ${ }^{1,3}$, Raquel Bailón ${ }^{1,3}$, Vaidotas Marozas ${ }^{4}$ \\ ${ }^{1}$ BSICoS group, Aragón Institute of Engineering Research, University of Zaragoza, Zaragoza, Spain \\ ${ }^{2}$ Department of Electrical Engineering (ESAT), STADIUS Centre for Dynamical Systems, Signal \\ Processing and Data Analytics, KU Leuven, Leuven, Belgium \\ ${ }^{3}$ CIBER en Bioingeniería, Biomateriales y Nanomedicina (CIBER-BBN), Zaragoza, Spain \\ ${ }^{4}$ Biomedical Engineering Institute, Kaunas University of Technology, Kaunas, Lithuania
}

\begin{abstract}
Finger and forehead pulse photoplethysmographic $(P P G)$ signals are compared as a surrogate for the electrocardiogram (ECG) in Heart Rate Variability (HRV) analysis during tilt table test. PPG signals are usually corrupted by motion artifacts. In this work, robust algorithms for pulse rate estimation have been applied. Classical time and frequency domain indices in the low frequency $(L F)$ and high frequency $(H F)$ bands have been estimated from pulse rate variability $(P R V)$ derived from both PPG signals. These PRV indices have been compared with those obtained from the reference $H R V$ derived from the ECG.

The relative error (median/interquartile range) between $P R V$ and HRV indices are comparable during early and later supine position in the forehead and finger signals (5.27/7.95\% vs $5.88 / 7.87 \%$ in the LF band, $6.84 / 13.23 \%$ vs 7.08/12.50\% in the HF band, 2.86/4.58\% and 3.17/4.43\% in the SDSD index during early supine position in the forehead and finger, respectively). The relative error indices estimated during the tilt were higher than during supine position, with slightly better performance in the forehead than in the finger (9.60/11.68\% vs 5.28/18.64\% in the LF band, $23.35 / 37.07 \%$ vs $35.94 / 81.95 \%$ in the HF band, $5.97 / 18.82 \%$ vs $12.71 / 49.03 \%$ in the SDSD index, during tilt in the forehead and finger, respectively). These results suggest that recordings on the forehead seem to provide better performance for the PRV analysis in nonstationarity environments.
\end{abstract}

\section{Introduction}

HRV analysis is a widely-used technique for the evaluation of the autonomic nervous system (ANS). Electrocardiography (ECG) has been a traditional method to determine the heart rate (HR), which is usually measured as the RR interval from the ECG.

Alternative technologies as photoplethysmography (PPG) require less complex and less costly sensors while also contains HR information related to blood volume changes, which is usually measured as the peak to peak interval of the corresponding PPG pulse. Given its simplicity, HR monitoring using PPG signals has become popular within the emerging wearables technologies for healthcare and fitness [1].

There are two basic techniques to acquire the PPG signal through a non-invasive pulse oximetry method: 1) transmission mode, where the light transmitted through the tissues is detected by a photodetector, and 2) reflectance mode, where the photodetector detects light that is backscattered from tissues [2].

The robustness of the pulse detection depends on the morphology of the PPG signal, where the main vulnerability of PPG signals are the motion artifacts caused by body movements. Hence, accurate PRV estimation in non-stationary and noisy environments is challenging.

In this paper, a comparison of HRV from ECG and PRV from reflection and transmission PPG during tilt test has been done to examine whether PPG signals acquired with different techniques can be used as a surrogate signal for ECG in HRV analysis, as has been shown in other studies [5], [6], [7]. Both techniques to acquire the PPG signal have been studied using signals recorded on the finger by transmission mode, and on the forehead by reflection mode. An extension study of the artifact detection algorithm [3] is presented in this work, and the pulse detector developed previously in [4] has been further adapted and extended to non-stationary intra-subjects conditions.

\section{Materials and Methods}

\subsection{Tilt Test Data and Preprocessing}

ECG and PPG data were simultaneously recorded from 18 subjects by Cardioholter6.2-8E78 (BMII, Lithuania). The sampling rate was $500 \mathrm{~Hz}$ for the ECG and $250 \mathrm{~Hz}$ for both PPG signals. For this study, a $500 \mathrm{~Hz}\left(f_{s}\right)$ resampling has been used for all signals. The protocol consisted of 
three- time intervals: 10 minutes in early supine position (Supine I-Early Supine), 5 minutes in head-up tilted (Tilt) and 5 minutes back to supine position (Supine II-Later Supine).

The database include: ECG lead II and four PPG signals, at two wavelengths, red and infrared, on the finger and the forehead. For all PPG signals, a high pass baseline filter with a cut-off frequency of $0.35 \mathrm{~Hz}$ has been applied. Besides, a low pass filter has been used with a cut-off frequency of $35 \mathrm{~Hz}$ for the transmission PPG signals and, $5 \mathrm{~Hz}$ for the reflection PPG signals, because forehead PPG signals are smoother and they may be affected by high frequency interference.

\subsection{Artifact detection}

The artifact detector based on Hjorth parameters presented in [3] has been applied. It is based on detecting when the PPG signal differs from an oscillatory signal using the first Hjorth parameter $\left(\mathrm{H}_{1}\right)$ as an estimation of the central frequency of the signal, and the second Hjorth parameter $\left(\mathrm{H}_{2}\right)$ as half of the bandwidth. For an intrasubject robustness analysis, a median adaptive filter has been implemented using a 4-minutes window length to define $\widehat{H_{1}(n)}$ and $\widehat{H_{2}(n)}$. A signal segment is considered as an artifact under the conditions (1) and (2), and that segment is discarded from the heart/pulse variability analysis.

$$
\begin{aligned}
& H_{2}(n)>\widehat{H_{2}(n)}+U_{2 u p} \\
& H_{1}(n)>\widehat{H_{1}(n)}+U_{1 \text { up }} \text { or } H_{1}(n)<\widehat{H_{1}(n)}-U_{1 \text { low }}
\end{aligned}
$$

Empirical thresholds derived from finger and forehead are defined in Table 1. It has been applied a stricter threshold in the reflection-based PPG signals because their morphology is characterized by smooth areas with higher possibility of noise presence.

Table 1. Artifact detector parameters

\begin{tabular}{cccc}
\hline & Finger & Forehead \\
\hline$U_{1 \text { up }}$ & & $1.4 \mathrm{~Hz}$ & \\
$U_{1 \text { low }}$ & & $1 \mathrm{~Hz}$ & \\
$U_{2 \text { up }}$ & $1.7 \mathrm{~Hz}$ & & $0.8 \mathrm{~Hz}$ \\
\hline
\end{tabular}

\subsection{PPG pulses detection}

An important point to stress is that during PRV analysis the accuracy in the fiducial points detections depends on the morphology of the PPG pulse waves. In this work, three fiducial points were computed: apex $\left(n_{A}\right)$, medium $\left(n_{M}\right)$ and basal $\left(n_{B}\right)$. The computation of these points in the pulse detector developed previously in [4] was modified in order to better suit for the smoother shapes of the reflection-based PPG signals. It detects the maximumupslope point of each PPG pulse $\left(n_{A}^{*}\right)$ by using a low pass differentiator filter and time-varying threshold.

The first one $\left(n_{A}\right)$ is set at the maximum point of the PPG signal $\left(x_{P P G}\right)$ within a $\tau_{A}$ ms-window, which was set as the median of five $n_{A}^{*}$ to $n_{A}^{*}$ intervals previously detected:

$$
n_{A}=\underset{n \in \tau_{A}}{\arg \max }\left\{x_{P P G}(n)\right\}
$$

Subsequently $n_{B}$ is set as the minimum within a $250 \mathrm{~ms}$ window ending at each $n_{A}^{*}$ :

$$
\begin{gathered}
n_{B}=\underset{n \in\left[n_{A}^{*}-0.25 f s, n_{A}^{*}\right]}{\arg \min }\left\{x_{P P G}(n)\right\} \\
\end{gathered}
$$

The $n_{M}$ is set as the point between $n_{A}$ and $n_{B}$ in which the amplitude has reached $50 \%$ of the maximum of the pulse amplitude as it was described in [4].

Fig. 1 shows the significant points of the $i_{\text {th }}$ PPG pulse for both PPG signals and the ECG reference.

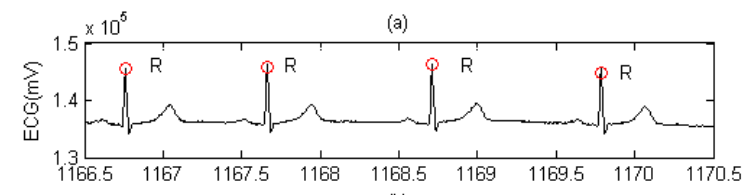

(b)

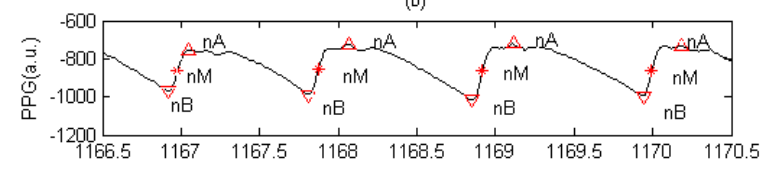

(c)

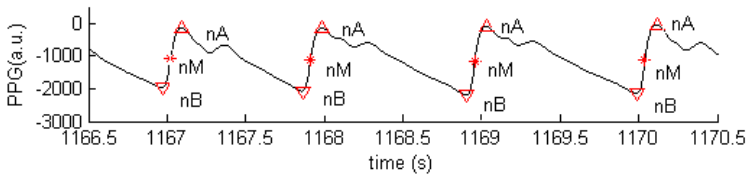

Figure 1. An example of (a) ECG, lead II, (b) reflection PPG signal, forehead, (c) transmission PPG signal, finger.

\subsection{Variability Analysis}

Classical time and frequency domain indices have been estimated during supine rest and head-up tilt from both HRV and PRV. Based on [8], the analysis of time domain was performed by means of standard deviation of $\mathrm{NN}$ intervals (SDNN), the standard deviation of the successive differences between adjacent NNs (SDSD), the square root of the mean of the squares of the successive differences between adjacent NNs (RMSSD), and the percentage of adjacent $\mathrm{NN}$ intervals with a difference duration greater than $50 \mathrm{~ms}$. The indices studied for the frequency domain are $\mathrm{LF}(0.04$ to $0.15 \mathrm{~Hz}), \mathrm{HF}(0.15$ to $0.4 \mathrm{~Hz}), \mathrm{LF}_{\mathrm{nu}}, \mathrm{HF}_{\mathrm{nu}}$ and $\mathrm{LF}$ to $\mathrm{HF}$ ratio.

HRV and PRV are analyzed from the detection of the QRS complexes from the ECG and from the $n_{M}$ fiducial point of each pulse from the PPG respectively. The maximum of the $\mathrm{R}$ wave is detected by using a waveletbased algorithm [9]. An instant heart/pulse rate signal $\mathrm{X}_{\mathrm{SRI}}(\mathrm{n})$ is obtained from both signals $(\mathrm{s} \in \mathrm{H}, \mathrm{P})$ using a generalization of the IPFM model [10] and spline interpolation. The signal $\mathrm{X}_{\mathrm{SRM}}(\mathrm{n})$ is defined as an estimation of the mean HR or PR by low-pass filtering with a cut-off frequency of $0.03 \mathrm{~Hz}$. HRV and PRV are obtained as the difference between the signal $x_{S R I}(n)$ and the signal $x_{S R M}(n)$.

The Welch periodogram [11] has been chosen as a non- 
parametric method of spectral estimation. Once the spectrum is estimated, the power of each frequency band is calculated by integrating the $x_{S R V}(n)$ spectrum into their respective frequency bands. In each band, the following indices have been computed: the power in low frequency band $\left(P_{L F}, P_{L F n u}\right)$, high frequency band $\left(P_{H F}, P_{H F n u}\right)$ and the LF to $\mathrm{HF}$ ratio $\left(R_{L F / H F}\right)$.

Classical time and frequency analysis have been performed in three intervals (Supine I, Tilt and Supine II). HRV and PRV indices have been calculated in 2-min windows, where stationarity is assumed. In case the criterium is not met, the window is shortened until there are no artifacts within the window. However, if the window length is shorter than 1 minute, that window is discarded from further analysis. The relative error in the PRV estimation is calculated for each index $I_{j}^{S R V}$ using the HRV signal as a reference according to:

$$
\mathrm{E}=100 \frac{I^{P R V}-I^{H R V}}{I^{H R V}}
$$

I: time and frequency domain indices

As result of the study we obtain the median/interquartile range values, which are calculated from the windows obtained among all subjects in each interval.

\section{Results}

Results obtained for forehead and finger infrared recordings using the fiducial point $n_{M}$ in the frequency domain and time domain are shown in tables 2 and 3, respectively. Table 4 shows the average percentage of discarded signal during the performance of the HRV and PRV analysis during each tilt test interval analyzed and, for each PPG recording technique studied in this paper, reflection and transmission respectively.

Table 2. Frequency domain PRV errors (median/interquartile range)

\begin{tabular}{|c|c|c|c|}
\hline & Index & $\begin{array}{l}\text { Relative Error } \\
\text { Forehead }(\%)\end{array}$ & $\begin{array}{c}\text { Relative Error } \\
\text { Finger }(\%)\end{array}$ \\
\hline \multirow{5}{*}{ 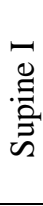 } & LF & $5.27 / 7.95$ & $5.88 / 7.87$ \\
\hline & $\mathrm{HF}$ & $6.84 / 13.2307$ & $7.08 / 12.50$ \\
\hline & $\mathrm{LF}_{\mathrm{nu}}$ & $-0.017 / 0.27$ & $-0.02 / 0.32$ \\
\hline & $\mathrm{HF}_{\mathrm{nu}}$ & $0.017 / 0.27$ & $0.021 / 0.33$ \\
\hline & $\mathrm{LF} / \mathrm{HF}$ & $-1.09 / 14.70$ & $-1.30 / 11.51$ \\
\hline \multirow{5}{*}{$\stackrel{=}{E}$} & LF & $9.60 / 11.68$ & $5.28 / 18.64$ \\
\hline & $\mathrm{HF}$ & $23.35 / 37.07$ & $35.94 / 81.95$ \\
\hline & $\mathrm{LF}_{\mathrm{nu}}$ & $-10.10 / 16.98$ & $-18.02 / 35.06$ \\
\hline & $\mathrm{HF}_{\mathrm{nu}}$ & $1.30 / 4.41$ & 1.88/11.97 \\
\hline & $\mathrm{LF} / \mathrm{HF}$ & $-12.55 / 18.34$ & $-18.19 / 48.60$ \\
\hline \multirow{5}{*}{ 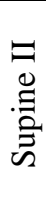 } & LF & $1.89 / 17.94$ & $3.23 / 10.34$ \\
\hline & $\mathrm{HF}$ & $6.56 / 53.70$ & $9.39 / 24.89$ \\
\hline & $\mathrm{LF}_{\mathrm{nu}}$ & $-5.90 / 28.03$ & $-3.21 / 27.76$ \\
\hline & $\mathrm{HF}_{\mathrm{nu}}$ & $0.1619 / 0.5264$ & $0.055 / 0.68$ \\
\hline & $\mathrm{LF} / \mathrm{HF}$ & $-6.11 / 30.47$ & $-3.26 / 28.41$ \\
\hline
\end{tabular}

Table 3. Time domain PRV errors (median/interquartile range)

\begin{tabular}{|c|c|c|c|}
\hline & Index & $\begin{array}{l}\text { Relative Error } \\
\text { Forehead }(\%)\end{array}$ & $\begin{array}{c}\text { Relative Error } \\
\text { Finger }(\%)\end{array}$ \\
\hline \multirow{5}{*}{ 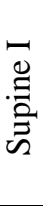 } & HRM & $0.004 / 0.07$ & $0.007 / 0.041$ \\
\hline & SDNN & $1.86 / 2.08$ & $1.90 / 2.09$ \\
\hline & SDSD & $2.86 / 4.58$ & $3.17 / 4.43$ \\
\hline & RMSSD & $2.86 / 4.59$ & $3.11 / 4.42$ \\
\hline & pNN50 & $0.044 / 6.16$ & $1.49 / 4.63$ \\
\hline \multirow{5}{*}{ 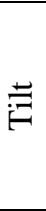 } & HRM & $0.024 / 0.12$ & $-0.033 / 0.55$ \\
\hline & SDNN & $3.37 / 3.75$ & $2.37 / 8.93$ \\
\hline & SDSD & $5.97 / 18.82$ & $12.71 / 49.03$ \\
\hline & RMSSD & $5.96 / 18.80$ & $12.72 / 48.94$ \\
\hline & $\mathrm{pNN} 50$ & $2.88 / 6.09$ & $4.91 / 30.69$ \\
\hline \multirow{5}{*}{ 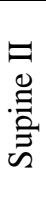 } & HRM & $-0.009 / 0.22$ & $-0.001 / 0.57$ \\
\hline & SDNN & $0.88 / 5.13$ & $2.04 / 3.84$ \\
\hline & SDSD & $7.30 / 9.53$ & $7.67 / 11.24$ \\
\hline & RMSSD & $7.29 / 9.59$ & $7.68 / 11.22$ \\
\hline & pNN50 & $1.94 / 5.21$ & $2.13 / 6.84$ \\
\hline
\end{tabular}

Table 4. Average time and percentage of artifacts

\begin{tabular}{crr}
\hline & Forehead & Finger \\
\hline Supine I & $72 \mathrm{sec}-11.9 \%$ & $98 \mathrm{sec}-16.33 \%$ \\
\hline Tilt & $58 \mathrm{sec}-19.3 \%$ & $83 \mathrm{sec}-27.67 \%$ \\
\hline Supine II & $60 \mathrm{sec}-20 \%$ & $60 \mathrm{sec}-20 \%$ \\
\hline
\end{tabular}

\section{Discussion}

PRV analysis during tilt table test from finger and forehead PPG signals is used to assess the changes in the autonomic nervous system as a surrogate of HRV analysis. The maximum $n_{A}$ is normally located at smooth zones where a low level of noise can significantly change its location. The minimum $n_{B}$ that could be used for PPG pulse detection depends on the morphology of the signal as it is shown in Figure 1. However, $n_{M}$ is located at the upward slopes of the PPG signal, which is an abrupt zone and therefore it is more robust against noise in all kind of PPG morphologies. Hence, it has been considered $n_{M}$ as the most accurate fiducial point for PRV analysis.

Time domain. Time domain indices derived from PRV present a small relative error during supine position, with values lower than $7 \%$. It has been shown that in short-term variability related indices, such as SDSD and RMSSD, the relative errors are higher during tilt than during supine interval. The global results suggest that PRV analysis could be used as a surrogate measurement of HRV analysis specially in the forehead PPG signals, with relative errors of $6 \%$ compared to $12 \%$ in the finger PPG signals.

Frequency domain. LF and HF components have been evaluated in both absolute and normalized (n.u) units. Table 2 shows higher relative errors in HF components during tilt position. This observation may be due to the low values obtained in the reference value within the HF band from the ECG, causing a significantly increase in the 
relative error value estimated during the performance of the $I_{j}^{S R V}$. Low relative errors indices indicate a strong relation between both signals, PRV and HRV, in LF and HF components during early and later supine position, with values lower than $10 \%(-1.89 / 17.94 \%$ vs $3.23 / 10.34 \%$ in the LF band, $6.56 / 53.70 \%$ vs $9.39 / 24.89 \%$ in the HF band during later supine position in the forehead and finger, respectively). A significant but weaker relation is shown on the indices derived during tilt, with relative errors slightly higher in the finger than the forehead within the HF components and LF/HF ratio $(-9.60 / 11.68 \%$ vs $5.28 / 18.64 \%$ in the LF band, $23.35 / 37.07 \%$ vs $35.94 / 81.95 \%$ in the HF band during tilt position in the forehead and finger, respectively).

In this study, the relative errors values obtained for the forehead signal are slightly better than the finger signal, especially during tilt position. In Table 4 , it can be observed the percentage of discarded signal during the performance of the PRV analysis, around $15 \%$ for all signals. The artifact presence in the transmission-based PPG signals is $20 \%$ higher than in the reflection-based PPG signals (190 sec vs $241 \mathrm{sec}$ of average discarded signal). These observations suggest that the accuracy of detecting the pulse depends on the morphology of the PPG signal according to the recording methodology and the different possible signal interferences or artifacts. Forehead signal is characterized by a lower signal intensity although it has been less contaminated by the presence of artifacts. Therefore, this study indicates that the recordings on the forehead could provide more reliable PRV information for non-stationarity conditions, while finger recordings should be further studied to verify and remove motion artifacts.

\section{Conclusions}

The purpose of this paper was to evaluate and compare the possibility of using PRV as an alternative measurement of the HRV signal during tilt test, when the PPG signal is recorded by a transmission sensor on the finger and by a reflection sensor on the forehead. It has been shown a higher accuracy in forehead pulse detection, which is characterized by a lower artifact interference. The relative errors values obtained during supine position are slightly better in the forehead than the finger as a surrogate measurement of HRV. In addition, forehead reflectionbased signal might be preferred for PRV analysis during standing position.

\section{Acknowledgements}

This work is supported by project TIN2014-53567-R of UZ, funded by MINECO, by Biomedical Research Networking Center on Bioengineering, Biomaterials and Nanomedicine (CIBER-BBN) of the Institute of Health
Carlos III, (ISCIII), by Grupo Consolidado BSICoS from DGA (Aragon) and European Social Fund (EU).

\section{References}

[1] Allen, John. Photoplethysmography and its application in clinical physiological measurement. Physiological measurement, 2007, vol. 28, no 3, p. R1.

[2] Tamura, Toshiyo, et al. Wearable photoplethysmographic sensors-past and present. Electronics, 2014, vol. 3, no 2, p. 282-302.

[3] Gil, Eduardo; Vergara, José María; Laguna, Pablo. Detection of decreases in the amplitude fluctuation of pulse photoplethysmography signal as indication of obstructive sleep apnea syndrome in children. Biomedical Signal Processing and Control, 2008, vol. 3, no 3, p. 267-277.

[4] Lazaro, Jesús, et al. Pulse rate variability analysis for discrimination of sleep-apnea-related decreases in the amplitude fluctuations of pulse photoplethysmographic signal in children. IEEE journal of biomedical and health informatics, 2014, vol. 18, no 1, p. 240-246.

[5] Gil, E., et al. Photoplethysmography pulse rate variability as a surrogate measurement of heart rate variability during nonstationary conditions. Physiological measurement, 2010, vol. 31, no 9, p. 1271.

[6] Dehkordi, Parastoo, et al. Pulse rate variability compared with heart rate variability in children with and without sleep disordered breathing. En Engineering in Medicine and Biology Society (EMBC), 2013 35th Annual International Conference of the IEEE. IEEE, 2013. p. 6563-6566.

[7] Jeyhani, Vala, et al. Comparison of HRV parameters derived from photoplethysmography and electrocardiography signals. En Engineering in Medicine and Biology Society (EMBC), 2015 37th Annual International Conference of the IEEE. IEEE, 2015. p. 5952-5955.

[8] Task Force, "Task Force of The European Society of Cardiology and The North American Society of Pacing and Electrophysiology. Heart rate variability: Standards of measurement, physiological interpretation, and clinical use". European Heart Journal, vol. 17, pp. 354.381, 1996.

[9] Martínez, Juan Pablo, et al. A wavelet-based ECG delineator: evaluation on standard databases. IEEE Transactions on biomedical engineering, 2004, vol. 51, no 4, p. 570-581.

[10] Mateo, Javier; Laguna, Pablo. Analysis of heart rate variability in the presence of ectopic beats using the heart timing signal. IEEE Transactions on Biomedical Engineering, 2003, vol. 50, no 3, p. 334-343.

[11] Welch, Peter. The use of fast Fourier transform for the estimation of power spectra: a method based on time averaging over short, modified periodograms. IEEE Transactions on audio and electroacoustics, 1967, vol. 15, no 2, p. 70-73.

Address for correspondence.

Elena Peralta,

Campus Rio Ebro, Universidad de Zaragoza, C/ Maria de Luna 1, 50018 Zaragoza (Spain)

elena.peralta.calvo@gmail.com 